\title{
10. INTELLIGENT ROBOTICS AND REMOTE SYSTEMS FOR THE NUCLEAR INDUSTRY
}

\author{
D.K. WEHE, J.C. LEE, W.R. MARTIN \\ Department of Nuclear Engineering, University of Michigan, Ann Arbor, MI 48109, USA
}

\author{
R.C. MANN and W.R. HAMEL \\ Oak Ridge National Laboratory, Oak Ridge, TN 37831, USA
}

and

\section{J. TULENKO}

Department of Nuclear Engineering. University of Florida, Gainsville, FL 32611, USA

\subsection{Introduction}

The nuclear industry has a recognized need for intelligent, multitask robots to carry out tasks in harsh environments. From 1986 to the present, the number of robotic systems available or under development for use in the nuclear industry has more than doubled [10-1]. Presently, artificial intelligence (AI) plays a relatively small role in existing robots used in the nuclear industry. Indeed, the lack of intelligence has been labeled the "Achilles heel" of all current robotic technology [10-2]. However, larger-scale efforts are underway to make the multitask robot more sensitive to its environment, more capable to move and perform useful work, and more fully autonomous via the use of AI. In this paper, we review the terminology, the history, and the factors which are motivating the development of robotics and remove systems; discuss the applications related to the nuclear industry; and, finally, examine the state of the art of the technologies being applied to introduce more autonomous capabilities. Much of this latter work can be classified as within the artificial intelligence framework.

\subsection{Motivation for using robotics in nuclear engineer-} ing

It is appropriate to begin our discussion of robotics and nuclear engineering by reviewing some related history and terminology. From a technical perspective, the application of robotics to nuclear problems is as old as nuclear research and power production itself. Early in the genesis of nuclear technology, radiation hazards forced the development of remotely operated equipment to protect human experimenters [10-3]. These remote techniques led to the rapid development of rather sophisticated human-controlled machines and manipulators [10-4]. This era was in the 1940's through the early 1960's when electronic systems were primitive compared to present microprocessor control technology. Except for sophisticated computer controls and microelectronics, the developments of this era can be reasonably classified as "robotics," but in the language of the day were termed as "remote systems." They involved complex mechanical and electromechanical systems and precision servomechanisms. The first such manipulators were developed in this era by Ray Goertz's famous team at the Argonne National Laboratory. Many of the concepts and principles they established remain valid and endorsed today, as well.

The term "robotics" became popular in the 1970's as the notion of a universal and programmable, automated manufacturing device became a practical reality due primarily to powerful and low-cost microprocessors [105]. These industrial robots can be thought of as versatile six-dimensional positioning machines designed to handle parts and tools. Interestingly, there are striking similarities between industrial robots and the master/ slave manipulators used for teleoperations in hazardous radioactive environments. These two fields have converged in recent years in virtually almost every aspect of 
functionality and performance [10-6]. Today, the two fields are nearly synonymous as a result of the crossfertilization in both research results and equipment applications.

The motivation for applying robotics and remote systems technology to nuclear engineering has evolved with advancing technologies. The original motivation, as discussed above, was to physically separate people from radioactive hazards. Of course, the definition of radiological hazards has changed dramatically over the years. Current regulations require that occupational radiation exposure be "as low as reasonably achievable". Another motivation is essentially building into a remotely operated system the ability to respond to unexpected or emergency events. Accidents like Three Mile Island and Chernobyl resulted in not only hazardous but, in some cases, lethal radiation conditions. In both accidents, robotic systems played critically important roles in postaccident surveillance and recovery operations $[10-7,-8]$. The final motivation to be discussed here is increased productivity in the operation of nuclear systems. In the proper context, robotic automation facilitates the automation of complex repetitious operations, resulting in greater accuracy and speed compared to equivalent manual operations. Robots are much better suited for precise and repetitious tasks than are humans. Due to current sensor and microelectronic technologies, effective automation of specific tasks in the complex nuclear engineering world is feasible and desirable.

These three motivations - hazard reduction, emergency response, and improved productivity - apply to all aspects of nuclear engineering systems, as well as the various steps in the nuclear fuel cycle: (a) mining operations, (b) enrichment, (c) fuel fabrication, and (d) fuel reprocessing. Reactor operations are also important and include fuel handling plus virtually every aspect of maintenance and operations of the nuclear primary systems within containment, including surveillance and inspection. They also apply to the increasingly important area of effective waste management including waste preprocessing and storage operations. The research environments that bore these technologies, including hot-cell operations, high energy particle accelerators, and fusion research machines, are also continuous sources of new applications.

\subsection{Robotics and remote technology history}

Teleoperators are machines that effectively project man's innate sensory and physical capabilities across
REMOTE CONTROL ENGINEERING (RCE) DEVELOPMENT ACTIVITIES INTENDED TO IMPROVE REMOTE MAINTENANCE TOOLS

TASK ORGANIZATION FOLLOWS BASIC SUBSYSTEM STRLCTLRE

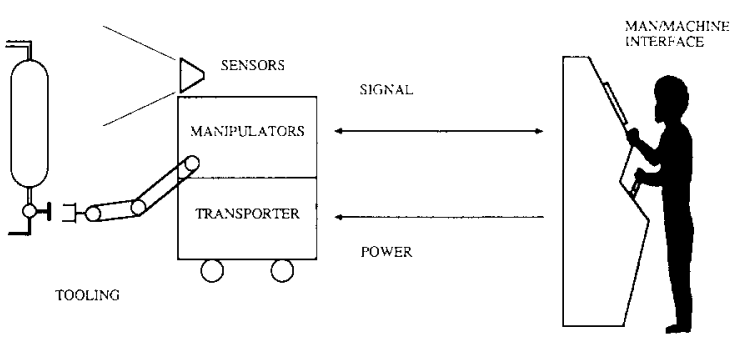

Fig. 10-1. Basic remote system structure.

distance and physical barriers [10-9]. Various remotely operated systems used in nuclear applications are correctly viewed as teleoperators. Remote maintenance systems, which usually have rather sophisticated handling capabilities are the most complex remotely operated systems in use today. Fig. 10-1 shows the basic elements generally included in a remote maintenance system. These subsystems serve the following basic functions: (a) human-machine interfacing, (b) signal and power transmission/storage, (c) mobility, (d) manipulation, and (e) remote sensory feedback.

Robot manipulators occur in many varieties, but they generally can be considered servo-controlled three-dimensional positioning and handling mechanisms. A historical summary of their evolution is shown in fig. 10-2. A typical kinematic arrangement incorporates three degrees of freedom in an upper and lower arm-like structure to position a three-dimensional wrist-like device which orients, or points, the hand or end-effector.

In the types of robots useful for nuclear engineering, the manipulation function involves the greatest technical complexity associated with system mechanization and serves the role of transfering the human operator's ability to handle objects, such as tools, into the remote environment. The fidelity of this telepresence phenomena is a dominant factor in the efficiency of the overall systems and is directly related to the dexterity and controllability of the robot manipulator system (and also to the quality of the sensory perception as well) [10-10]. Another key aspect of robots is the issue of mobility. An effective manipulation system is of little value unless it can be transported to the work site of interest. The achievement of high mobility is a contextdependent issue. Mobility on the factory floor is signifi- 
cantly simpler than versatile mobility within a nuclear reactor containment area. Over the years, a spectrum of wheeled, tracked, and legged mobile platforms have been developed for emergency response, explosive ordinance disposal, and nuclear accident response. A synopsis of these can be found in ref. [10-11]. A family of impressive walking robots has been developed by the Odetics Corporation. These robots hold great promise for operation within very complex topologies, and nuclear applications are a particular area of emphasis [10-12]. As discussed in ref. [10-13], the mechanical man which would approximate anthropomorphism, to the extent that technology will allow, will weigh at least an order of magnitude more than a real man. This is because of the poor weight efficiency of electrical actuators and chemical battery power storage. The advances of the past $10 \mathrm{yr}$ in robotics technology have been substantial, but many important challenges remain. As section 10.5 discusses, introducing $\mathrm{AI}$ into these machines is a dominant issue in the industry today.

\subsection{Robotics applications in nuclear engineering}

Nuclear engineering applications of robotics generally result in unique requirements. This results from the basic characteristics of the work task environments and general character of the operational environments. In industrial robotics applications, one can generally impose constraints, principally in the form of jigs and fixtures which accommodate the limitations of the manipulators, sensors, and end-effectors. Jigs and fixtures essentially restrain the location and position/ orientation of objects of interest to be compatible with the robot. The research trend in manufacturing robotics is to incorporate sensors like computer vision. With this feature, the work task environment need not be as structured, which is expensive and restrictive. Most nuclear engineering applications, in contrast to industrial robotics, involve the remote operations and repair of highly complex systems which represent highly unstructured work task environments - much like those
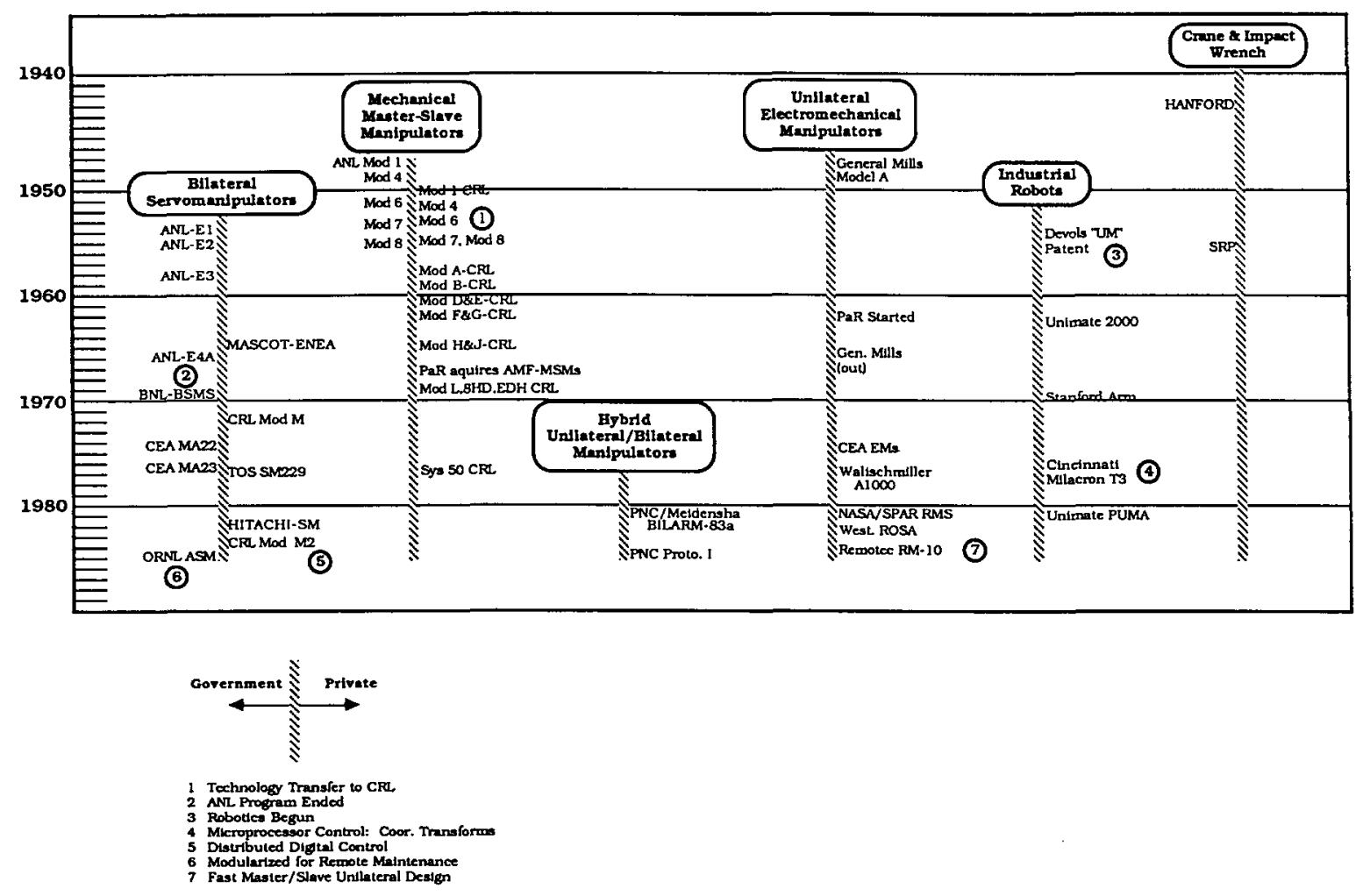

Fig. 10-2. History of manipulator developments. 
that we as humans deal with routinely (e.g., petrochemical processing plants or nuclear reactor containments). Due to the complexity and uncertainty of the nuclear work task environment, great effort is made to effectively integrate human supervision and control into teleoperator-robotic systems. In this way, the human is functionally positioned to compensate for the machine as needed. Human skills for visual scene interpretation and for decision making using incomplete data are still superior to the most advanced AI implementations, although the gap is narrowing. To exemplify the characteristics of nuclear engineering applications, refer to ref. [10-14] for a recent and comprehensive sampling of this type of work. Three particularly fertile areas for future robotic applications are nuclear fuel reprocessing, nuclear power reactor operations, and fusion research applications. The latter two are discussed below.

\subsubsection{Nuclear power reactor operations}

Aside from fuel handling operations and steamgenerator tube sheet repairs, the advantages of robotics have not been adequately exploited in power reactor systems. A number of studies have shown that if robotic solutions can reduce reactor outage times and occupation radiation exposures, huge economic savings are possible since off-line power reactors represent losses of $\$ 1,000,000$ per day to utilities. Identified application areas include auxiliary building systems and the nuclear supply systems within containment. Tasks cover a very wide range of inspection and maintenance tasks involving small components like radiation detectors to very large components such as primary pump seals. The complexity of the typical reactor containment environment is a difficult problem and was a major motivation for the development of the walking robot, Odex III, built by the Odetics Corporation for the Electric Power Research Institute (EPRI). In addition, the Department of Energy is funding a long-range research program involving four major universities and Oak Ridge National Laboratory (ORNL) to develop intelligent mobile robots for advanced fission reactors.

\subsubsection{Fusion research applications}

Fusion systems such as the Joint European Torus and the planned Compact Ignition Tokamak should yield plasma operating conditions which will introduce activation products and attendant radioactive containments. Consequently, the fusion domain is already being examined for robotic and remote technology applications [10-14]. The exterior of the typical tokamak in- volves handling operations similar to reactors and reprocessing. Inside the vacuum vessel, the work space involves toroidal geometry, has limited access ports and is surrounded by delicate, precise components such as graphite insulating tiles. Redundant degrees of freedom manipulators, which can "snake" around the toroid, are under development. Ultimately, like fission reactors, it is expected that robotics and remote systems technology will play a central role in achieving economical and safe fusion power production.

As shown above, the existing applications of robotics within nuclear engineering have had limited onboard intelligence. The robot or remote system has primarily mimicked the physical motions of the operator or operated as a "dumb" industrial robot. But mobile, teleoperated vehicles have recognized drawbacks [10-15] and can impose undesirable control burdens on the operator. As a result, intensive research efforts are underway to enhance the mobility and sensory and manipulative capabilities of mobile robots. In addition, significant strides are also being made in introducing "intelligence" into the robot, i.e., a degree of autonomy, through the application of AI techniques. Some of the major research robots developed for the nuclear industry and which are pioneering this field are summarized below.

Robin is the Savannah River Laboratory's walking (legged) robot built by Odetics Corporation. Monitoring and control are performed by a teleoperator console which is connected by a fiber optic cable to the robot. The complete control of the robotic system is by a hierarchical structure of microcomputers. The microcomputers coordinate the teleoperator and all basic movement algorithms and interactions with real-time commands from sensory devices (the touch sensors on the articulators) [10-16].

The Surveyor system, produced by Automation Technology Corporation, is a remotely operated, tetherless, track vehicle which uses a computer-based operation in both a supervisory control station and the vehicle. In addition, it possesses two onboard, highspeed, low-power microcomputers which control motor operation, data acquisition and supervision in the vehicle, and data formatting and display at the control station. With the addition of intelligence, some tasks originally performed by the operator in Surveyor I can now be handled by this robot. Surveyor II performs such tasks as stair climbing and placement of inspection optics autonomously [10-17].

The FramAtome Resources Development Division has developed a robot that basically is a computer that interacts with the outside world. A command center 
controls the overall system operations, including movement of the vehicle, manipulator arms, and instruments; supervision and performance of tasks; and simulation and diagnostics [10-18].

Developed by the Japanese reactor vendor Toshiba, the Intelligent Maintenance Robot possesses clover-leaf wheels that enable it to climb over obstacles and up and down stairs. The manipulator has nine degrees of freedom and a visual unit which has sufficient recognition of passages, walls, and obstacles to position the robot automatically. It also has the capability of recognizing the object on which is in performing work. The manipulator is easily remotely operated through a display unit providing three-dimensional color graphics [10-19].

Toshiba Corporation and a team from Japan's Tokyo University have jointly developed a robot known as Amooty. It can move automatically inside a plant according to a memorized "map". Amooty can obtain its position by using a television camera to recognize low reflective squares placed along its route. Its relative location is determined by projecting a laser at two symbols and measuring the distance on the basis of size and profiles of the television image of the reflection beam. If there are obstacles in the robot's path, it can recognize and avoid them or automatically change its course to take another path to achieve the same goal. The individual subsystems can be directed independently or jointly by a microcomputer, thus displaying substantial onboard intelligence [10-20].

Surbot, produced by Remote Technology Corporation for the Nuclear Regulatory Commission, is controlled by a unique combination of teleoperated and robotic technology called telerobotics. It is possible to teach Surbot a specific path and to perform a set of surveillance activities within a room. Future entries into the room could be totally autonomous. The robot arm, tower, television camera pan/tilt, and other sensor actuators show similar intelligent properties [10-21].

The number of mobile robots in existence today which could prove useful to the nuclear industry is actually much larger than the six robots mentioned above. Ref. [10-22] presents a compendium of over 90 mobile robots useful for hazardous environments. However, a much higher level of onboard intelligence will be required before these robots can be employed in practical, routine, general-purpose field applications.

\subsection{The role of $\mathrm{Al}$ in robotics}

Although the robots described in section 10.4 display more intelligent behavior than teleoperated vehicles, major advances in sensory perception and various aspects of machine intelligence are required to implement fully autonomous or self-controlled intelligent robots. The remainder of this paper will focus on the following areas of autonomous robotics research which are generally considered within the AI framework: decision making intelligence, vision, multisensor integration, world modeling and knowledge base manipulation, and navigation and path planning. The reader is also referred to ref. [10-23] for a pragmatic view of robotic research needs and ref. [10-24] for a more philosophical view of $\mathrm{AI}$ applications in robotics.

\subsubsection{Decisionmaking intelligence}

Successful operation and deployment of a robot in an ill-structured nuclear power plant environment depends markedly on its ability to perform, in unsupervised mode, tasks involving a high degree of intelligence. Robotics has been defined as the "intelligent connection of perception to action" [10-24]. Whether explicitly termed artificial intelligence or not, robotic systems are being imbued with increasing amounts of onboard processing capabilities.

As a "simple" example, the robotic intelligence may be based on an expert system, involving a knowledge base and an inference system. The expert system must have the ability to update, in modular structure, its knowledge base and the rules for accessing and utilizing the knowledge base. The expert system may utilize both heuristic and simulation-based knowledge in a symbiotic manner. Simulation-based knowledge is derived from physical models of the system and comparison of the effects predicted from simulating potential causes to the actual effects observed. Pattern recognition algorithms can be applied to establish key features characterizing a given problem space. These features can then be used to establish causal relationships - a task normally associated with learning.

In summary, the research goal is to provide the tools that allow the computer to infer and deduce knowledge using sensory-derived data as input. This intelligence provides the vehicle with the capability to make some decisions autonomously and relieves the human operator from continuous and tedious low-level control responsibilities. In addition, the top-level control, which determines which tasks to perform and in what order, will almost certainly take the form of an AI expert system.

\subsubsection{Vision}

Computer vision is perhaps the most important automated sensor for robotics. It is beyond the scope of this 
paper to review this area in detail. Simplistically viewed, the process begins with a TV-generated digital image, upon which operations are performed, such as edge detection and smoothing. Recognition of three-dimensional objects, and then entire scenes, is a difficult research issue - particularly if the lighting is not optimal or changing, or if the objects are allowed to move. Other research issues involve identifying partially obscured or overlapping objects, building three-dimensional maps from a series of individual images - either using stereo cameras or through the motion of a single camera, and reading or interpreting gauges and alphanumeric characters. Image understanding involves mixing general expert system heuristics with vision images in order to make decisions about the content of the image. Using knowledge enables one to expand the capabilities of vision systems to solve less constrained problems and to attack a more general class of vision problems. Indeed, much of the current vision research is aimed at making smarter systems, i.e., more reliable and flexible systems that do not require total redesign for slight changes in the problem. Thus, the application of AI techniques has been critical in the development of robotic vision systems.

\subsubsection{Navigation and path planning}

Intelligent navigation and task planning are other examples of difficult research issues that have benefited from the application of AI techniques. When a robot must move an arm or a mobile platform must move, the robot must be able to reason about space so it can avoid objects and reach the desired target. The task requires both local knowledge obtained from sensors and global knowledge from a model of the environment. This knowledge can then be processed to search for paths and movement strategies to achieve an acceptable trajectory. The use of AI techniques expedites a solution to several types of these search problems. Fast expert systems have also been reported that permit real-time navigation in simple, unstructured environments [10-25]. Other scientists are working on developing a more general set of top-level heuristics to be used for autonomous navigation [10-26]. Higher-level controls use reactive reasoning and planning which include features of beliefs, desires, and intentions associated with achieving goals [10-27]. Other approaches use multiple layers of interacting control philosophies which interact with each other in a behavioral, rather than functional, manner [10-28]. The reader is referred to Refs. [10-29] through [10-32] for other samples of similar approaches being applied to the navigation problem.
10.5.4. Integration and interpretation of uncertain sensory data

One of the prerequisites for intelligent behavior in robotic systems is the ability to generate consistent representations of the sensed environment. Because of the inherent limitations of practical sensor technology, this is impossible on the basis of any single sensor domain. Hence, robotic systems are being equipped with an increasing number of different sensors that supply partly redundant information. Multisensor integration (MSI) designates the task of combining data and information from these various sensors such that a consistent world model, i.e., a model free of contradiction, can be generated on the basis of which decisions concerning navigation, manipulation, etc. can be made. A panel of experts, at a recent workshop on research needs for intelligent machines, has identified MSI as an issue of highest priority [10-33].

The task is highly complex because of several circumstances related to the diversity of sensors. Depending on the physics underlying a particular sensor the amount of time required to acquire data varies among different sensors. The amount of time needed to perform quantitative analysis of sensor data and extract features also depends on the nature of the sensor. For example, analysis of digital images is generally more time-consuming than the analysis of one-dimensional signals. This introduces scheduling and synchronization problems in a dynamic environment. The confidence associated with a sensor measurement depends on the situation in the environment, e.g., a sonar sensor tends to underestimate distances to room corners and cannot detect open spaces between obstacles whose distance from each other is smaller than the width of the ultrasound beam. It follows that there should be a sensor hierarchy that depends on the environment as well as on the particular mission of the robotic system. Thus, any mechanism for MSI must have access to the knowledge base describing the world model, and flow of information between different sensor domains must be possible at all stages in the data and information processing. Finally, a consistent representation of the environment on the basis of the available sensors is not guaranteed. A method for MSI must recognize such a situation and be able to deal with this uncertainty.

In advanced robotic systems with a high degree of autonomy for certain tasks, MSI needs to be accomplished under real-time constraints. Real-time performance is dictated by the speed at which the robot must operate in order to accomplish a task and be economically viable. Thus, advanced computing architectures 
(discussed below) are being considered for implementing methods of MSI. And because of the variety of the problems that an intelligent robot will be required to handle, more general MSI routines must be developed. It is in this area, such as developing sensor hierarchies, that AI-based approaches show the greatest potential.

\subsubsection{World modeling (knowledge based representations)}

A knowledge-based robotic system must be able to store and recall the information it needs to analyze, so as to be able to make decisions in an autonomous fashion. The essential part of a knowledge-based robotic expert system is a world model which acts as a central repository of information about the robot and the environment. This data base provides the robot with both knowledge about and a description of its environment. The two common approaches are the geometric CAD/ CAM model and the voxel model. The CAD/CAM model is self-explanatory, while the voxel approach breaks all of space into increasingly fine boxes. While the geometric model provides an inherent physical connectivity between components and is computationally more compact, the voxel approach is currently favored for its compatibility with the MSI problems discussed in section 10.5.4.

The organization of this knowledge becomes very important for both timely and efficient utilization of this data. Since all the details do not have the same importance for all possible tasks, it is essential that the model (data base) have several levels of abstract representation. The robot may use multiple levels in its knowledge-based reasoning depending on the complexity of the task.

The world modeling process produces a set of entities that are rich enough in associated attributes that they can be analyzed by an expert system using appropriate techniques in order to autonomously carry out commands. A key item of a world model is that it is easy to comprehend, to maintain, and to integrate into the sensor-based knowledge-gathering systems of a robot.

\subsubsection{Man-machine interface}

The man-machine interface is a critical feature of an intelligent robotic system that allows humans to interact with the robot. The man-machine interface must facilitate the cooperative problem-solving relationship between a human and a machine which, together, compose the available knowledge base and knowledge-reasoning capability. In the robotic man-machine inter- face, where man is attempting to project his capabilities over long distances, the man-machine interface is quite complex.

As the capability for robotic systems to perform more autonomously increases, the task allocation between man and machine becomes more involved. The same knowledge-reasoning capability that allows robots to act autonomously can be expanded for the dynamic optimization of tasks between man and machine in a changing environment. Of particular difficulty, however, is the case where the human and machine dynamically share tasks in an unexpected manner. For instance, the human may unexpectedly interrupt a task execution, perform some action telerobotically, and then wish to hand control back to the robot. This sharing of cognitive tasks represents a new area of research called intelerobotics [10-34] and has its roots in early AI work [10-35].

\subsubsection{Advanced computer architectures and algorithms}

A limiting factor for eventual practical utilization of robots in real-world problems is the sheer computational burden for carrying out the many and diverse tasks of the robot, including vision, multisensor integration, optimal and adaptive control, simulation-based and reactive reasoning, motion prediction, and simulation. In order to have this computational capability on board as well as affordable, extensive use will need to be made of advanced parallel architectures, which offer the potential of low cost and high performance.

There are numerous parallel architectures being developed in this country, by academic groups as well as industry. There are basically two classes of parallel architectures, shared-memory and distributed-memory architectures. Advanced controllers for robotics will likely be one of two configurations: general purpose distributed memory MIMD (multiple instruction/ multiple data) machines or networks of shared memory multiprocessor systems. Several computers representative of each category are currently being marketed by various vendors, and the list is likely to grow. Current shared-memory architectures (such as the high-end represented by Cray or ETA, or even the more modest Alliant) are easier to program but are too costly and too large for real robotics applications.

Thus, it seems that distributed-memory architectures show high potential for robotics applications [10-36, -37]. There are several candidate architectures, two of which are hypercubes and neural networks. The hypercube architecture of cube order $N$ utilizes $2^{N}$ processors and can be visualized as a cube of dimension $N$. Each 
processor is connected to $N$ processors, resulting in at most $N$ "hops" to allow one processor to communicate to another processor in the array. Hypercubes have demonstrated an extremely attractive cost/performance ratio for scientific computing. Because this class of parallel processors was the first to be commercially developed to a large extent (e.g., Intel NCUBE, Floating Point Systems, and Ametek to date), a great deal of experience has been obtained with them. Supercomputer performance (for example, Cray speeds) has been reported for applications in scientific computation for single-board hypercube computers, indicating the potential of this class of computer architecture for real problems.

The autonomous mobile robot HERMIES, developed in the ORNL CESAR Program, is equipped with an onboard hypercube of dimension 4 (16 nodes) manufactured by the NCUBE Corporation. The system handles sensory information processing and navigation in a dynamic environment [10-37]. An example of multiple processor arm control is given in ref. [10-38].

Recently, there has been a resurgence of interest in artificial neural networks for computing [10-39]. In contrast to hypercube architectures, where one attempts to map the application onto the architecture (e.g., via an algorithm), a neural network in essence involves programming the architecture to fit the application. An example of this paradigm is an adaptable robot with two arms and three vision cameras which uses neural networks to learn eye arm coordination through practice [10-40]. But despite successful applications of neural network to problems in pattern recognition [10-41] and combinatorial optimization [10-42], major theoretical and technical advancements are required to take full advantage of the potential these massively parallel architectures offer and to produce corresponding hardware and software capable of achieving the performance of biological systems for complex sensory and cognitive tasks.

\subsection{Conclusions}

The nuclear industry has historically been the motivating force in the development of robotics for hazardous environments. The demand for more autonomous and capable mobile robots has spurred large-scale research efforts into the ways in which these machines can be imbued with more inherent intelligence.

Thus, AI techniques have become essential tools to the development of advanced-generation robots. Indeed, the fifth generation robot, which is projected to reach maturity between 2005 and 2010, is expected to be a strictly AI-controlled, walking machine [10-43]. Although the problems dealing with the complexities of the real world are substantial, the magnitude of the benefits dictate that this technology will continue to be developed and applied.

\section{References}

[10-1] H.B. Meieran, Mobile robots gain momentum, Nuclear Engineering International 32, 393 (1987) pp. 34-35.

[10-2] N.S. Rajaram, Artificial Intelligence - The achilles heel of robotics and manufacturing, Robotics Engineering (January 1986) p. 10.

[10-3] R. Goertz, Some work on manipulator systems at ANL: Past, present, and a look at the future, Proceedings of 1964 Seminars on Remotely Operated Special Equipment, USAEC Report CONF-641120 (May 1964).

[10-4] W. Hamel and M. Feldman, The advancement of remote systems technology: Past perspectives and future plans, Proceedings of 1984 ANS Topical Meeting on Robotics \& Remote Handling in Hostile Environments, Gatlinburg, Tennessee, April 1984.

[10-5] J. Engelberger, Robotics in practice, AMACOM, 1980.

[10-6] W. Hamel and H. Martin, Robotics-related technology in the nuclear industry, Proceeding of SPIE, Robotics and Robot Sensing Systems, 442 (August 1983).

[10-7] Y. Adamov and Y. Yegorov, Development of robotic systems for nuclear applications including emergencies, Proceedings of 1987 ANS Topical Meeting on Robotics \& Remote Handling in Hostile Environments, Pasco, Washington, April 1987.

[10-8] H. Alexander-Remote systems applications at Three Mile Island Unit 2, Proceedings of 1984 ANS Topical Meeting on Robotics \& Remote Handling in Hostile Environments, Gatlinburg, Tennessee, April 1984.

[10-9] E. Johnsen and W. Corliss, Teleoperators and human augmentation, NASA Report NASA SP-5047 (1967).

[10-10] S. Babcock et al., Advanced manipulation for autonomous mobile robots, Proceedings of 1987 ANS Topical Meeting on Robotics \& Remote Handling in Hostile Environments, Pasco, Washington, April 1987.

[10-11] F. Gelhaus and H. Meieran, Currently available mobile teleoperators and their applicability to radiological emergencies, Proceedings of Workshop on Requirements for Mobile Teleoperators for Radiological Emergency Response and Recovery, ANL Report ANL/EES-TM-291 (June 1985).

[10-12] T. Bartholet et al., Robot applications for nuclear power plant maintenance, EPRI-NP-3941 (March 1985).

[10-13] S. Babcock and W. Hamel, Manipulator technology: The critical element of useful autonomous working machines, International Congress on Intelligent Systems, Amsterdam, The Netherlands, December 1986. 
[10-14] Proceedings of 1987 ANS Topical Meeting on Robotics \& Remote Handling in Hostile Environments, Pasco, Washington, April 1987.

[10-15] J.S. Byrd and J.J. Fisher, Expert robots in nuclear plants, Proceedings of the ANS International Topical Meeting on Artificial Intelligence and Other Innovative Computer Applications in the Nuclear Industry, Snowbird, Utah, August 31-September 2, 1987 (Plenum Press, New York, 1988).

[10-16] T.G. Bartholet, Odetics makes great strides, Nuclear Engineering International 31, 381 (1986) pp. 43-44.

[10-17] R.K. Simmons, Smart systems for routine jobs, Ibid. pp. 41-42.

[10-18] T.L. Irving, FramAtome develops a mobile vehicle for maintenance, Ibid. 32, 393 (1987) pp. 51-52.

[10-19] U. Uesugi and H. Okano, Toshiba looks to save skilled labor, Ibid. pp. 38-39.

[10-20] A. Cruickshank, Amooty, a stair climbing intelligent maintenance robot, Ibid. 30, 367 (1985) p. 36.

[10-21] J.K. White, Surbot makes surveillance safer, Ibid. 31, 381 (1986) pp. 36-41.

[10-22] H.B. Meieran, A compendium on mobile robots used in hazardous environments, EPRI NP-5060 (May 1987).

[10-23] D. Nitzan, Development of intelligent robots: Achievement and issues, IEEE Journal of Robotics and Automation, RA-1, 1(March 1985).

[10-24] M. Brady, Artificial intelligence and robotics, Artificial Intelligence 26 (1985) pp. 79-121.

[10-25] C. Weisbin, G. deSaussure, D. Kammer, A real-time expert system for an autonomous mobile robot, Computers In Mechanical Engineering (September 1986) p. 12.

[10-26] R. Chattergy, Some heuristics for the navigation of a robot, International Journal of Robotics Research 4, 1 (Spring 1985).

[10-27] M.P. Georgeff and A.L. Lansky, Reactive reasoning and planning, Proceedings of the 1987 Conference of the American Association for Artificial Intelligence, Seattle, Washington, p. 677.

[10-28] R.A. Brooks, A robust layered control system for a mobile robot, IEEE Journal of Robotics and Automation, RA-2, 1 (March 1986).

[10-29] J.L. Crowley, Navigation for an intelligent mobile robot, Ibid, RA-1, 1 (March 1985).

[10-30] M.P. Georgeff and A.L. Lansky, Reactive reasoning and planning, Proceedings of the 1987 Conference of the American Association for Artificial Intelligence, Seattle, Washington.

[10-31] Y. Ichikawa and N. Ozaki, Path planning and map updating for mobile robot, Proceedings of SICE '87, Hiroshima, Japan, July 15-17, 1987.

[10-32] B.J. Kuipers and T.S. Levitt, Navigation and mapping in large-scale space, AI Magazine 9, 2 (Summer 1988) p. 25.

[10-33] Research Needs in Intelligent Machines, Proceedings of a workshop, November 1984, prepared for the Center for Engineering Systems Advanced Research, ORNL, Oak Ridge, Tennessee, (Oklahoma State University, Stillwater, Oklahoma).

[10-34] L. Conway, R. Volz, M. Walker, Tele-autonomous systems: Methods and architectures for intermingling autonomous and telerobotic technology, Proceedings of the 1987 IEEE International Conference of Robotics and Automation, March 30, 1987.

[10-35] F. Hayes-Roth, D.W. Waterman, D.B. Lenat, eds., Building Expert Systems (Addison-Wesley, Reading, Massachusetts, 1983).

[10-36] J. Barhen and J. Palmer, The hypercube in robotics and machine intelligence, Computers in Mechanical Engineering, 4 (1986).

[10-37] B.L. Burks et al., Autonomous navigation, exploration, and recognition using the HERMIES-IIB robot, to appear in IEEE Expert.

[10-38] R.A. Brooks, J. Connell, A. Flynn, A mobile robot with onboard parallel processor and large workspace arm, Proceedings of the 1986 Conference of the American Association for Artificial Intelligence.

[10-39] IEEE Proceedings of the First International Conference on Neural Networks, San Diego, California, June 1987.

[10-40] I. Peterson, Robots with a lot of nerve, Science News 131 (June 6, 1987) p. 362.

[10-41] T. Kohonen, Self-organization and associative memory (Springer-Verlag, New York, 1984).

[10-42] J.R. Einstein and J. Barhen, Intelligent operating systems for autonomous robots: Real-time capabilities on a hypercube super-computer, Proceedings of 1987 ANS Topical Meeting on Robotics \& Remote Handling in Hostile Environments, Pasco, Washington, April 1987.

[10-43] T.M. Knasel, Mobile robotics - State of the art review, Robotics 2 (1986) pp. 149-155. 